\title{
Love and Rayleigh phase-velocity maps, 5-40 s, of the western and central USA from USArray data
}

\author{
G. Ekström ${ }^{\mathrm{a}}$ \\ ${ }^{a}$ Lamont-Doherty Earth Observatory of Columbia University, Palisades, NY 10964 \\ email: ekstrom@ldeo.columbia.edu; phone: +1(845)365-8427; FAX: +1(845)365-8150
}

\begin{abstract}
Continuous data recorded on more than 1600 USArray seismic stations operating in the western and central US between 2006 and 2012 are used to map phase velocities of Love and Rayleigh waves at short periods (5-40 s) using a noise-correlation technique. Vertical and transverse records for all station pairs separated by less than $600 \mathrm{~km}$ are cross correlated in 4-hourlong segments, and the resulting spectra are stacked for the time period of station operation. Dispersion curves are determined from the locations of zeros in the real component of the correlation spectra using a method based on that of Aki (1957). Phase-velocity maps expanded on a $0.25^{\circ}$-by- $0.25^{\circ}$ pixel grid are estimated by inversion of the phase-velocity measurements. Comparison with predicted phase-velocity maps based on the crustal model CRUST2.0 combined with the mantle model ND08 shows good agreement at the longer periods. Strong slow anomalies $(>25 \%)$ in the short-period maps are geographically correlated with basins and regions of thick sedimentary cover. The strength of these anomalies is not well predicted by existing crustal-velocity models.
\end{abstract}

Keywords: Surface-wave dispersion, Noise tomography, Earth's crust 
1

2

3

4

5

6

7

8

9

\section{Introduction}

One of the unexpected, useful applications of the data recorded by the Transportable Array (TA) of USArray is the mapping of crustal structure using surface-wave signals extracted from seismic noise. It is worth noting that none of the three community articles that formulated the case for constructing USArray (Ekström et al., 1998; Levander et al., 1999; Meltzer et al., 1999) mentions continent-scale crustal mapping as a goal, much less achieving this goal by analyzing noise. Because of the relatively large station spacing $(\sim 70 \mathrm{~km})$, the TA was, at the beginning, mainly viewed as useful for constraining mantle lithospheric and deep Earth structure. This perception gradually changed in the early 2000s when several studies demonstrated the practical possibility of extracting robust structural signals from crosscorrelations of seismic noise recorded at two stations. Pioneering papers include those by Shapiro and Campillo (2004), who showed the presence of wavetrains with Rayleigh wave dispersion in noise cross-correlation functions for stations separated by tens and several hundreds of kilometers, and by Shapiro et al. (2005) and Sabra et al. (2005), who demonstrated the possibility of using dispersion measurements obtained from cross-correlation functions for tomographic imaging of phase velocity across southern California. With its regular 2-D-grid geometry and 18- to 24-month-long sliding deployments, the TA turns out to be ideal for surface-wave mapping with noise cross-correlation functions. The last several years have seen rapid growth in research testing and exploiting this capability.

It is apparent from numerous tomographic-mapping experiments that both Love and Rayleigh wave signals over a broad range of relatively short 
periods (a few seconds to tens of seconds) can be extracted from crosscorrelation functions from station pairs at TA offsets $(\geq 70 \mathrm{~km})$ (e.g., Shapiro et al., 2005; Sabra et al., 2005; Yao et al., 2006; Lin et al., 2008). Mapping of phase velocities at these periods provides new constraints on $P$ and $S$ velocities in the crust, as well as on anisotropy. In combination with other seismic measurements, such as those provided by receiver-function analysis, knowledge of surface-wave dispersion properties allows better-constrained mapping of crustal velocity structure and depths of horizontal interfaces such as the Moho and the top of the crystalline basement.

Most recent studies that have used noise cross correlation to extract dispersion information for tomographic inversion start from the premise that, for a sufficiently diffuse background-noise wavefield, the cross-correlation function should approach the inter-station Green function (e.g., Lobkis and Weaver, 2001; Snieder, 2004; Sánchez-Sesma and Campillo, 2006). This motivates and justifies the use of standard analysis methods for extracting surface-wave group- and phase-velocity measurements from the time-domain cross-correlation function; in essence treating it as a point-source seismogram. In an earlier paper (Ekström et al., 2009), we took an alternative approach, and returned to the treatment by Aki (1957) of the properties of the noise cross-correlation spectrum. In Aki's approach, the underlying assumption is that the cross-correlation function is dominated by contributions from fundamental-mode surface waves. With this assumption, dispersion properties can be directly inferred from the cross-correlation spectrum using algorithms that are straightforward to automate. In our 2009 study, we demonstrated the performance of this spectral approach, and documented 
high similarity of our tomographic results with other early results derived from USArray data.

In this paper, we extend the method of Ekström et al. (2009) and apply it systematically to the seismic data recorded on USArray. The first objective is the development and presentation of a large data set of inter-station phasevelocity measurements derived from analysis of cross-correlation spectra. The second and main objective is the determination of baseline Love and Rayleigh phase-velocity maps over the period range 5-40 s for the western two thirds of the conterminous US. The third objective is an evaluation of the maps by qualitative and quantitative comparison with an existing continent-scale crustal model and maps of sediment thickness.

We assess the uncertainties and the internal consistency of the data set by comparisons of measurements of similar paths, and by the ability of the phase-velocity models to predict the observations. We do not, in this contribution, explore the potential effects on measurements or on derived maps of the non-uniform distribution of noise sources, or other uncertainties associated with the noise cross-correlation method. We also limit the tomographic analysis to a standard ray-theory treatment. By publishing the data used in the inversions, we hope to facilitate other uses of these observations. The phase-velocity maps presented here, and the similarities and differences that we document between the maps and predictions from existing crustal models, identify directions for future work. In particular, we expect the models and data presented here to be useful for developing better models of the elastic structure of deep sedimentary basins. 


\section{Theory and Methods}

Theoretically, the cross correlation of a diffuse and equipartitioned elastic wavefield sampled in two locations takes the form of the elastic Green function between the two locations (e.g., Snieder, 2004; Sánchez-Sesma and Campillo, 2006). Over the last decade, this property has been explained theoretically and explored in a number of papers. The phenomenon has also been exploited in many practical applications, leading to the establishment of the new discipline of Ambient Noise Tomography (ANT) (e.g., Bensen et al., 2007). The extent to which the conditions for recovering the true Green function are present in any given real experiment or data set is generally unknown, but from a wealth of studies it is clear that, at a minimum, the cross-correlation function calculated for two seismic stations contains the dispersive properties of fundamental-mode surface waves traveling from one station to the other. This property was first used in seismology by Aki (1957).

Aki (1957) considered the correlation of stochastic, horizontally propagating waves recorded at two stations and derived expressions for the azimuthally averaged cross spectra for dispersive and polarized waves. A key result is his equation 42 ,

$$
\bar{\rho}\left(r, \omega_{0}\right)=J_{0}\left(\frac{\omega_{0}}{c\left(\omega_{0}\right)} r\right),
$$

which states that the azimuthally averaged normalized cross spectrum $\bar{\rho}\left(r, \omega_{0}\right)$ for a receiver separation $r$ and frequency $\omega_{0}$ varies as $J_{0}$, the Bessel function of the first kind, where $c\left(\omega_{0}\right)$ is the phase velocity between the receivers at frequency $\omega_{0}$. Aki correlated observed zero crossings in $\bar{\rho}\left(r, \omega_{0}\right)$ with zeros of $J_{0}$ to determine the dispersion. In Part 3 of his 1957 paper Aki also 
argued, on the basis of similarities in the observed correlation functions derived from station pairs oriented at different azimuths, that the azimuthally averaged cross spectrum $\bar{\rho}\left(r, \omega_{0}\right)$ could be replaced in the analysis by the cross-correlation spectrum $\rho_{i j}(\omega)$ obtained between two individual stations $i$ and $j$. This argument is also the basis for most modern implementations of ANT, including ours.

Our measurement method follows the general approch of Aki (1957) and builds on our earlier implementation of the spectral method (Ekström et al., 2009). In the first step of the data processing, we collect long-period (LHchannel) continuous data from all stations of the USArray TA, the USArray Reference Network, and a few additional stations. The data are processed in 4.5-hour-long, half-hour overlapping, tapered time windows, with offsets of 4 hours, so that 6 records result for each component and each day. The instrument responses are deconvolved from the original records, and the data are stored as 4-500 s band-pass-filtered displacement spectra $u(\omega)$. We construct cross-correlation spectra for the pair of stations $i$ and $j$ as

$$
\rho_{i j k}(\omega)=\frac{u_{i k}(\omega) u_{j k}^{*}(\omega)}{\sqrt{u_{i k}(\omega) u_{i k}^{*}(\omega)} \sqrt{u_{j k}(\omega) u_{j k}^{*}(\omega)}},
$$

for each 4-hour data window $k$ that is common to the two stations. We calculate vertical cross correlations by cross correlation of the vertical-component records, and transverse cross correlations by first constructing the component transverse to the interstation great-circle direction using the two horizontal components of each seismometer, and then cross correlating the resulting transverse records. 
The individual spectra are summed to create stacked spectra,

$$
\rho_{i j}^{S}(\omega)=\sum_{k=1}^{N_{i j}} \rho_{i j k}(\omega),
$$

where $N_{i j}$ is the total number of 4-hour-long records available for a station pair, and $\rho_{i j}^{S}(\omega)$ is the stack. We do not apply whitening or other non-linear filtering to the data, and do not exclude time windows with earthquake signals. Strong earthquake signals do not dominate the stacked spectra because the contributions are normalized at the level of the 4-hour-long records, and the typical stack is built from more than 2500 records. This approach to signal conditioning and data selection is different from that typically employed (e.g., Bensen et al., 2007) and from our earlier work (Ekström et al., 2009), and was selected based on the outcome of experiments showing minimal differences in spectral dispersion measurements or final phase-velocity models using the different signal-conditioning schemes.

In the second step of the processing, the stacked cross-correlation spectra $\rho_{i j}^{S}(\omega)$ are analyzed to determine the locations of zero crossings in the real part of the correlation spectrum. Figure 1 shows the real and imaginary parts of the transverse correlation spectrum for stations ISA-CI and 109C-TA and the time-domain representation of the spectrum. The non-zero imaginary part of the spectrum is a manifestation of the asymmetry present in the time domain. The focus on the real part of the spectrum in our analysis is equivalent to considering only the part of the time-domain signal that is symmetric with respect to the zero-offset time. To reduce the number of spurious zero crossings when the amplitude of the spectrum is small, we smooth the raw spectrum by applying a running average with a width of approximately one 
third of the separation of zero crossings in the real part of the spectrum. The zero crossings are identified by the running index $n$ and denoted $\omega_{n}^{Z}$, with odd $n$ corresponding to crossings from positive to negative spectral amplitude, and even $n$ corresponding to crossings from negative to positive amplitude. The zeros are stored together with information concerning the amplitude of the local spectral extrema adjacent to each zero. These amplitudes are used in subsequent processing as indicators of the quality of the zero identification and its frequency.

In the third step of the processing, the zero crossings are analyzed to construct continuous dispersion curves. Figure 2 illustrates this step. Association of each observed zero crossing $\omega_{n}^{Z}$ with zeros of $J_{0}$ leads to estimates of phase velocity

$$
c_{m}\left(\omega_{n}^{Z}\right)=\frac{\omega_{n}^{Z} r}{z_{n+2 m}},
$$

where $m$ takes the values $0, \pm 1, \pm 2, \ldots$, indicating the number of missed or extra zero crossings in the observed spectrum. At long periods $(T \geq 25 \mathrm{~s})$, the correct $m$ is inferred by requiring that the phase velocity fall in a realistic range. Dispersion curves are constructed separately for odd and even $n$ by connecting the identified zeros with line segments. At shorter periods, the appropriate $m$ for each zero is selected on the basis of the range and smoothness of the dispersion curve defined by the values $c_{m}\left(\omega_{n}^{Z}\right)$ across frequencies $\omega_{n}^{Z}$. An automated search algorithm is used to track the dispersion curve from long periods to short periods, extending it by one zero at a time. When the choice of which Bessel function zero (which $m$ ) to associate with the next observed zero becomes ambiguous, or no smooth dispersion curve can be constructed, the extension of the dispersion curve to shorter periods 
is abandoned. The two dispersion curves (odd and even) are then sampled at discrete periods between $5 \mathrm{~s}$ and $40 \mathrm{~s}$, and the average of the two values is stored as the path-specific phase-velocity observation $c_{i j}$ between stations $i$ and $j$.

In the fourth step of the analysis, we determine maps of isotropic phase slowness across the footprint of USArray using a standard least-squares tomographic method. We parameterize the slowness maps in terms of $N$ latitudelongitude pixels and denote the slowness in each pixel $n$ by $p_{n}$. The distance along the great-circle path between stations $i$ and $j$ is written $X_{i j}$, and the fractional path length in each pixel is $X_{i j}^{n}$. We rewrite the observed phase velocity as an observed travel time, $\tau_{i j}=\frac{X_{i j}}{c_{i j}}$, and construct the corresponding model travel time as

$$
\tau_{i j}^{P}=\sum_{n}^{N} X_{i j}^{n} p_{n},
$$

where only those $X_{i j}^{n}$ that lie along the inter-station path will be non-zero. We form the misfit function

$$
\chi^{2}=\sum_{i j}^{K}\left(\frac{\tau_{i j}-\tau_{i j}^{P}}{\sigma_{i j}}\right)^{2}
$$

where the summation is over the $K$ station pairs $i j$ for which an observation is included at a particular period, and $\sigma_{i j}$ is an estimate of the observational uncertainty. We then determine $p_{n}$ by solving the damped least-squares problem

$$
\min \left(\chi^{2}+\nu R^{2}\right)
$$

where $\nu$ is a smoothing coefficient and $R^{2}$ is the roughness of the slowness 
variations. We calculate $R^{2}$ as

$$
R^{2}=\sum_{n}^{N}\left[\sum_{m}^{4}\left(\frac{p_{n}-p_{m}}{d_{n m}}\right)^{2}\right]
$$

where the index $m$ refers to the pixels north, south, east, and west of the $n$th pixel, and $d_{n m}$ is the distance between pixel centers.

\section{Analysis and Results}

We apply the measurement technique to USArray station data recorded between January 2006 and December 2012, corresponding to 1630 different stations. We stack data for all station pairs separated by less than $600 \mathrm{~km}$, leading to approximately 300,000 station pairs, with each stacked spectrum corresponding to, on average, $~ 3000$ four-hour-long correlation windows, or approximately 17 months of continuous data. Love and Rayleigh dispersion curves are extracted from the transverse and vertical stacks, respectively, and the dispersion curves are sampled at eleven discrete periods: 5, 6, 8, 10 , $12,15,20,25,30,35$, and $40 \mathrm{~s}$. Automatic selection based on the quality of the derived dispersion curves leads to an initial data set of approximately three million phase-velocity measurements for the two wave types and eleven periods.

The initial data set is trimmed by considering the wave period and interstation distance. Competing effects must be balanced in the data selection. On the one hand, it is desirable to include short paths in the inversion because they provide the best constraints on small-scale structure. On the other hand, measurements of short paths have greater relative errors, and the performance of the spectral method for station offsets on the order of a wavelength 
is not well known. Similarly, long paths provide useful extra path coverage and integral constraints on the slowness model, but may be more strongly affected by non-ray-like behavior. Uncertainties in the measurements at short periods for long paths are also larger as a result of difficulties in tracking the zero crossings that define the dispersion curve. Given these considerations, and the abundance of available data, we make a conservative selection based on distance such that $X_{\min }=2 \lambda$ and $X_{\max }=\max (5 \lambda, 200 \mathrm{~km})$, where $X_{\min }$ and $X_{\max }$ are the minimum and maximum inter-station distances and $\lambda$ is the wavelength, calculated using the period and a velocity of $4 \mathrm{~km} \mathrm{~s}^{-1}$. At the shortest periods, $X_{\max }$ is set to $200 \mathrm{~km}$ in order to ensure that a sufficient number of observations are included. At the longest periods $X_{\max }$ is also limited by the $600-\mathrm{km}$ overall maximum distance of the correlation-function data set. The resulting data set is summarized in Table 1 .

Before inversion, we investigate the internal consistency of the selected data by comparing phase-velocity measurements on long paths with the corresponding measurement constructed by combining two shorter paths. Consider stations $a, b$, and $c$ with inter-station distances $X_{a b}, X_{b c}, X_{a c}$ and phase-velocity measurements $c_{a b}, c_{b c}$, and $c_{a c}$. With $a c$ the longest distance, we denote the phase velocity predicted from $c_{a b}$ and $c_{b c}$ as $c_{a c}^{\prime}$, which we calculate as

$$
c_{a c}^{\prime}=\frac{X_{a b}+X_{b c}}{X_{a b} / c_{a b}+X_{b c} / c_{b c}} .
$$

To ensure rough great-circle alignment of the stations we apply the geometrical selection criteria $X_{a b}+X_{b c}<X_{a c}<1.1\left(X_{a b}+X_{b c}\right), X_{a b}>X_{a c} / 4$, and $X_{b c}>X_{a c} / 4$. Figure 3 shows the resulting scatter plot of $c_{a c}$ versus $c_{a c}^{\prime}$ for 85,620 path combinations that satisfy these criteria for Rayleigh waves at 
$10 \mathrm{~s}$ period. The correlation coefficient is 0.94 and the standard deviation of the distribution of differences $c_{a c}-c_{a c}^{\prime}$ is $0.05 \mathrm{~km} \mathrm{~s}^{-1}$, reflecting good agreement. No systematic offset between the two phase velocities is observed, nor is there a detectable deviation from a slope of 1.0 in the correlations. The standard deviations at other periods fall in the range $0.03-0.14 \mathrm{~km} \mathrm{~s}^{-1}$, with the largest value found for Love waves at $5 \mathrm{~s}$.

As is evident from Figure 3, the data set contains outliers. To suppress the influence of these outliers on the final models, we adopt a two-step inversion scheme with additional data weighting applied in the second step. In the first step, all of the data are included in the inversion with travel-time uncertainties $\sigma_{i j}$ corresponding to a uniform phase-velocity uncertainty in the raw measurement $c_{i j}$. The model is parameterized on a relatively coarse $0.5^{\circ}$-by- $0.5^{\circ}$ grid. This ensures that each pixel is sampled by a large number of rays, and that the small number of outliers have limited influence on the resulting model.

The misfit of the data to the predictions from the coarser model is then used to identify the outliers, and to adjust their associated uncertainty. Specifically, with $\delta c_{i j}$ denoting the data misfit following the inversion and $\sigma_{c}$ denoting the standard deviation of the misfit, we multiply uncertainties for observations that deviate by more than two standard deviations by the factor $\left(1+2\left(\frac{\delta c_{i j}}{\sigma_{c}}-2\right)\right)$. This effectively gives those data that were not well fit in the initial inversion less influence on the model derived in the second inversion.

In the second step of the inversion, the model is parameterized on a finer $0.25^{\circ}$-by- $0.25^{\circ}$ pixel grid. The Love and Rayleigh slowness models are 
derived by inversion as in equation (7). The damping factor $\nu$ is selected based on a combination of objective and subjective criteria. In general, less damping is required at short periods, for which the signal strength is high compared with the data uncertainties. At long periods, the damping results in relatively smooth models. The differences between the initial models and the second-iteration models are small: at all periods the correlations are $>0.97$. However, the two-step process effectively eliminates very-small-scale (single pixel) anomalies that can result from isolated erroneous measurements for short paths.

The slowness models that result from the second inversion are the main results of this study. In the presentation and discussion of the models, we convert phase slowness to phase velocity. Table 1 summarizes the number of data, average velocities, and the reduction in data variance provided by the maps.

\section{Discussion}

Figures 4 and 5 show our results for Love and Rayleigh waves in terms of phase-velocity variations at $5 \mathrm{~s}, 10 \mathrm{~s}, 20 \mathrm{~s}$ and $40 \mathrm{~s}$. At long periods ( $\geq 20 \mathrm{~s}$ ) the most prominent characteristic is the large-scale division of the continent into a slow western half and a faster eastern half, well documented in numerous earlier seismological studies (e.g., Romanowicz, 1979; Godey et al., 2004; Nettles and Dziewoński, 2008) and generally associated with the contrast between the stable cratonic plate interior in the east and the recently tectonically deformed boundary of the North America plate in the west. At short periods $(<20 \mathrm{~s})$, the velocity variations are strong and typically very 
localized. The velocity distributions around the areal means (the zero values in the maps) are not symmetric: very strong and concentrated slow anomalies are common while fast anomalies tend to be broad and less strong.

Most of the dramatic features seen in the short-period maps can be associated with specific, well-known geologic features. For example, in the state of Wyoming alone, distinct slow anomalies can be associated with the Powder River, Big Horn, Gros Ventre, River, Sand Wash, Hanna, and Denver Basins; and fast anomalies with the Bighorn Uplift, Wind River Mountains, Laramie Range, and Sierra Madre Mountains (e.g., Peterson and Smith, 1986). A more subtle and complex feature extending from Lake Superior to Kansas in both the Love and Rayleigh wave maps can be associated with the surface expression of the mid-continent rift. The largest-scale slow anomaly seen coherently in all maps at periods shorter than $20 \mathrm{~s}$ is located along the Gulf Coast, extending from the border with Mexico to the panhandle of Florida, with the most extreme slow velocities located in southern Texas, Louisiana and Mississippi.

To investigate the extent to which the phase-velocity maps are consistent with knowledge of the elastic structure of North America, we make comparisons with predictions based on a three-dimensional seismic-velocity model of the crust and mantle. It is evident that the short-period maps reflect shortwavelength variations in seismic velocities of the shallow crust that have not yet been mapped uniformly across the area of interest. We choose to make our predictions using the global model of the crust CRUST2.0 (Bassin et al., 2000) because it provides a consistent representation of the thicknesses and intrinsic properties of the crust across the area. A drawback of this model is 
its two-degree spatial parameterization, which does not allow the representation of some of the smaller features observed in the maps. We therefore do not focus on agreement between individual features, but rather summarize the overall correlation and discuss the ranges of anomalies that are observed and predicted.

To account also for large-scale mantle heterogeneity, we calculate the predictions for the combination of CRUST2.0 and mantle model ND08 (Nettles and Dziewoński, 2008), a radially anisotropic shear-velocity model of the mantle beneath North America embedded in a lower-resolution global model. The mantle model ND08 was derived using corrections for CRUST2.0. We also calculate the predictions for a simpler model with CRUST2.0 on top of the 1-D PREM mantle (Dziewonski and Anderson, 1981).

For the crust-and-mantle model, the correlations are positive at all periods (Figure 6), and high $(>0.7)$ at the longest periods. For neither Love nor Rayleigh waves does the crust-only model predict the long-period maps well, reflecting the fact that the long-wavelength patterns seen in Figures 4 and 5 result mainly from mantle heterogeneity. At short periods, the two models make very similar predictions, and are moderately well $(\sim 0.5)$ correlated with the observed maps, consistent with the shallow sensitivity of short-period surface waves and the accuracy of CRUST2.0.

To investigate the nature of the misfit, we compare the range of predicted dispersion curves for the crust-mantle model with the observed dispersion. Figure 7 shows the dispersion curves predicted for the study area calculated on a one-degree grid. To represent the corresponding observations, we calculate the median phase velocity for the study area at each period and identify 
the full range of values; we plot these together with the predicted dispersion curves. We observe consistency between the observed ranges and the models at faster velocities and at the longest periods. However, for both Love and Rayleigh waves, we observe many phase velocities that are significantly slower than predicted by CRUST2.0, most likely reflecting much lower shear velocities in some sedimentary basins. To illustrate this further, we show in Figure 8 dispersion observations and predictions for two locations: one in northern Minnesota $\left(47^{\circ} \mathrm{N}, 95^{\circ} \mathrm{W}\right)$ and one in southern Mississippi $\left(31^{\circ} \mathrm{N}\right.$, $\left.91^{\circ} \mathrm{W}\right)$. The agreement is very good for the Minnesota location, with both Love and Rayleigh waves well predicted even at short periods. Here, the phase velocities at $5 \mathrm{sec}$ essentially reflect the intrinsic $v_{P}$ and $v_{S}$ of the crystalline basement exposed at the surface. The Mississippi location displays extremely slow phase velocities at short periods, and very large disagreement with the predictions. The disagreement here cannot easily be attributed to the scale of the mapped anomalies, since similarly slow velocities extend inland from the coast at least $200 \mathrm{~km}$, and along the coast for several hundred $\mathrm{km}$. To bring the predicted dispersion closer to the observations, lower shear velocities and a thicker sedimentary layer are likely to be needed, as the anomalies are severely underpredicted for both Love and Rayleigh waves between $5 \mathrm{~s}$ and $15 \mathrm{~s}$.

We assess the relationship between short-period phase velocities and the sedimentary cover by comparing our phase-velocity maps with maps of sediment thickness provided by Mooney and Kaban (2010). We calculate the median phase velocity, and the range of the central quartiles, for pixels corresponding to different sediment thicknesses. Figure 9 shows the results for 
Love and Rayleigh waves at 5-s period. Love and Rayleigh phase velocities slower than $2.0 \mathrm{~km} \mathrm{~s}^{-1}$ are the norm for sediment thicknesses in excess of $10 \mathrm{~km}$, and for Love waves the median speed corresponding to these thicknesses is close to $1.5 \mathrm{~km} \mathrm{~s}^{-1}$. Such slow speeds are clearly not commonly predicted using existing models of the crust (Figure 7). It should be noted that the extreme slow velocities imaged at 5-s period in deeply sedimented areas may challenge assumptions in the analysis, such as the single-mode assumption inherent in the measurement technique, and the ray-theoretical formulation used in the imaging. The degree to which this may bias the imaged velocities is unclear. However, both overtone interference and nonstraight ray paths are likely to lead to overestimates of the velocity using our assumptions; true velocities may therefore be even slower than we have imaged here.

\section{Conclusions}

We have developed a method for cross correlating continuous long-period seismograms and extracting Love and Rayleigh wave phase-velocity information from the stacked cross-correlation spectra. The method is largely automated, allowing the collection of large data sets of uniform quality.

As in many automated measurement procedures, the greatest challenges are associated with automating choices in the analysis and quality selection. The construction of dispersion curves by connection of zero crossings in the spectra is well suited for a computer algorithm because each connection alternative can be evaluated based on a combination of quantitative merits, such as dispersion-curve smoothness or the plausibility, based on prior informa- 
tion, of a particular phase-velocity measurement. Though not explored here, a possible extension of the algorithm would involve iterating on the association of the zeros and the construction of dispersion curves using successively updated predictions from the derived phase-velocity models.

The automated measurement method generates a small number of bad observations, and we have chosen to suppress these outliers rather than applying more complicated data-selection criteria. The initial use of a slightly coarser model grid leads to oversampling of most pixels and corresponding dilution of the influence of outlying observations, which can then be downweighted in subsequent inversion with a finer grid. The dense and nearly uniform coverage provided by USArray inter-station paths makes this approach straightforward to implement, and we were easily able to verify that the downweighting of outliers had the anticipated effect. In applications with more variable spatial coverage, this simple two-step approach may be less effective.

The derived phase-velocity maps explain the new dispersion data well. At the longest periods, the large-scale anomaly patterns are consistent with previously mapped mantle heterogeneity. At short periods, the qualitative agreement between localized velocity anomalies and surface-geologic features is striking, but significant misfit is apparent between the observed velocities and corresponding predictions based on CRUST2.0. In particular, both Rayleigh and Love waves exhibit extremely slow velocities across deep sedimentary basins and along the heavily sedimented Gulf Coast, with the observations lying well outside the range of the predictions. Additional work is needed to reconcile these surface-wave velocities with other relevant ob- 
servations, such as depth to crystalline basement and measured sediment velocities. We speculate that while the $v_{P}$ structure of many of these regions is known, $v_{S}$ may not be as well determined, and may be overestimated. The slow surface-wave phase velocities could therefore be a manifestation of high $v_{P} / v_{S}$ ratios in the sediments to great depth.

Incorporation of phase-velocity constraints derived from noise tomography in models of the crust will not only improve understanding of shallow structures, but is also important for purposes of mantle imaging. While the sensitivity of long-period surface waves is small at shallow depths, the heterogeneities are strong, and may therefore still impart a significant signal.

The cross-correlation-function stacks $\left(\rho_{i j}^{S}(\omega)\right)$, the phase-velocity measurements $\left(c_{i j}\right)$, and the final slowness maps $\left(p_{n}\right)$ described here are made available at the IRIS DMC in conjunction with this publication, as well as at www.ldeo.columbia.edu/ ekstrom.

\section{Acknowledgments}

I thank Meredith Nettles, the Guest Editor, and two anonymous reviewers for useful comments that helped improve the manuscript. I am grateful to everyone involved in the deployment and operation of USArray. The consistent and high quality of the data from the array made the research here possible. I also thank the IRIS DMC for providing simple and efficient access to these data. This research was supported by the EarthScope Program of the National Science Foundation, grant EAR-0952285. 


\section{References}

Aki, K., 1957. Space and time spectra of stationary waves with special reference to microtremors. Bull. Earthquake Res. Inst. Univ. Tokyo 35, $415-456$.

Bassin, C., Laske, G., Masters, G., 2000. The current limits of resolution for surface wave tomography in North America. EOS, Trans. AGU 81, Abstract S12A-03, Fall Meeting Suppl.

Bensen, G.D., Ritzwoller, M.H., Barmin, M.P., Levshin, A.L., Lin, F., Moschetti, M.P., Shapiro, N.M., Yang, Y., 2007. Processing seismic ambient noise data to obtain reliable broad-band surface wave dispersion measurements. Geophys. J. Int. 169, 1239-1260.

Dziewonski, A.M., Anderson, D.L., 1981. Preliminary reference Earth model. Phys. Earth Planet. Int. 25, 297-356.

Ekström, G., Abers, G.A., Webb, S.H., 2009. Determination of surface-wave phase velocities across USArray from noise and Aki's spectral formulation. Geophys. Res. Lett. 36, L18301.

Ekström, G., Humphreys, E.D., Levander, A., 1998. USArray — probing the continent. IRIS Newsletter 16, 2-9.

Godey, S., Deschamps, F., Trampert, J., Snieder, R., 2004. Thermal and compositional anomalies beneath the North American continent. J. Geophys. Res. 109, B01308. 
Levander, A., Humphreys, E.D., Ekström, G., Meltzer, A.S., Shearer, P.M., 1999. Proposed project would give unprecedented look under North America. EOS, Trans. Am. geophys. Un. 80, 245-251.

Lin, F.C., Moschetti, M., Ritzwoller, M.H., 2008. Surface wave tomography of the western United States from ambient seismic noise: Rayleigh and Love wave phase velocity maps. Geophys. J. Int. 173, 281-298.

Lobkis, O.I., Weaver, R.L., 2001. On the emergence of the Green's function in the correlations of a diffuse field. J. Acoust. Soc. Am. 110, 3011-3017.

Meltzer, A., Rudnick, R., Zeitler, P., Levander, A., Humphreys, E.D., Karlstrom, K., Ekström, G., Carlson, R., Dixon, T., Gurnis, M., Shearer, P., van der Hilst, R., 1999. USArray initiative. GSA Today 9, 8-10.

Mooney, W.D., Kaban, M.K., 2010. The North American upper mantle: Density, composition, and evolution. J. Geophys. Res. 115, B12424.

Nettles, M., Dziewoński, A.M., 2008. Radially anisotropic shear velocity structure of the upper mantle globally and beneath North America. J. Geophys. Res. 113, B02303.

Peterson, J.A., Smith, D.L., 1986. Rocky Mountain paleogeography through geologic time, in: Peterson, J.A. (Ed.), Paleotectonics and sedimenation in the Rocky Mountain region, United States. Amer. Assn. of Petroleum Geologists. volume 41, pp. 3-19.

Romanowicz, B., 1979. Seismic structure of the upper mantle beneath the United States by 3-dimensional inversion of body wave arrival times. Geophys. J. Roy. Astron. Soc. 57, 479-506. 
Sabra, K.G., Gerstoft, P., Roux, P., Kuperman, W.A., Fehler, M.C., 2005. Surface wave tomography from microseisms in Souther California. Geophys. Res. Lett. 32, L14311.

Sánchez-Sesma, F.J., Campillo, M., 2006. Retrieval of the Green's function from cross correlation: The canonical elastic problem. Bull. Seism. Soc. Am. 96, 1182-1191.

Shapiro, N.M., Campillo, M., 2004. Emergence of broadband Rayleigh waves from correlations of the ambient seismic noise. Geophys. Res. Lett. 31, L07614.

Shapiro, N.M., Campillo, M., Stehly, L., Ritzwoller, M.H., 2005. Highresolution surface-wave tomography from ambient seismic noise. Science $307,1615-1618$.

Snieder, R., 2004. Extracting the Green's function from the correlation of coda waves: A derivation based on stationary phase. Phys. Rev. E 69, 046610 .

Yao, H., van der Hilst, R.D., de Hoop, M.V., 2006. Surface-wave array tomography in SE Tibet from ambient seismic noise and two-station analysis - I. Phase velocity maps. Geophys. J. Int. 166, 732-744. 


\begin{tabular}{rrrrccccc}
\hline & & & \multicolumn{3}{c}{ Love waves } & \multicolumn{3}{c}{ Rayleigh waves } \\
\cline { 5 - 9 } Period & $X_{\text {min }}$ & $X_{\max }$ & $N$ & $c$ & $\zeta$ & $N$ & $c$ & $\zeta$ \\
sec & $\mathrm{km}$ & $\mathrm{km}$ & & $\mathrm{km} \mathrm{s}^{-1}$ & & & $\mathrm{~km} \mathrm{~s}^{-1}$ & \\
\hline 5 & 40 & 200 & 23,240 & 3.02 & 0.95 & 22,324 & 2.93 & 0.93 \\
6 & 48 & 200 & 25,461 & 3.18 & 0.95 & 25,530 & 3.01 & 0.93 \\
8 & 64 & 200 & 25,999 & 3.38 & 0.95 & 26,616 & 3.12 & 0.94 \\
10 & 80 & 200 & 23,833 & 3.49 & 0.93 & 24,054 & 3.19 & 0.92 \\
12 & 96 & 240 & 35,975 & 3.58 & 0.92 & 36,214 & 3.25 & 0.91 \\
15 & 120 & 300 & 53,322 & 3.68 & 0.90 & 56,998 & 3.35 & 0.87 \\
20 & 160 & 400 & 47,522 & 3.82 & 0.83 & 73,979 & 3.51 & 0.90 \\
25 & 200 & 500 & 62,340 & 3.95 & 0.76 & 111,948 & 3.65 & 0.88 \\
30 & 240 & 600 & 74,425 & 4.05 & 0.73 & 164,872 & 3.75 & 0.87 \\
35 & 280 & 600 & 62,328 & 4.14 & 0.67 & 150,445 & 3.83 & 0.84 \\
40 & 320 & 600 & 48,596 & 4.21 & 0.60 & 128,754 & 3.88 & 0.81 \\
\hline
\end{tabular}

Table 1: Data set and inversion results. The distance range used at each period $\left(X_{\min }\right.$ and $\left.X_{\max }\right)$; the number of observations $(N)$ used in the inversion, the average phase velocity $(c)$ and the variance reduction $(\zeta)$ at each period and for each wave type. The variance-reduction calculation refers to phase-velocity misfit with respect to the best-fitting uniform-velocity model, and includes outliers. 

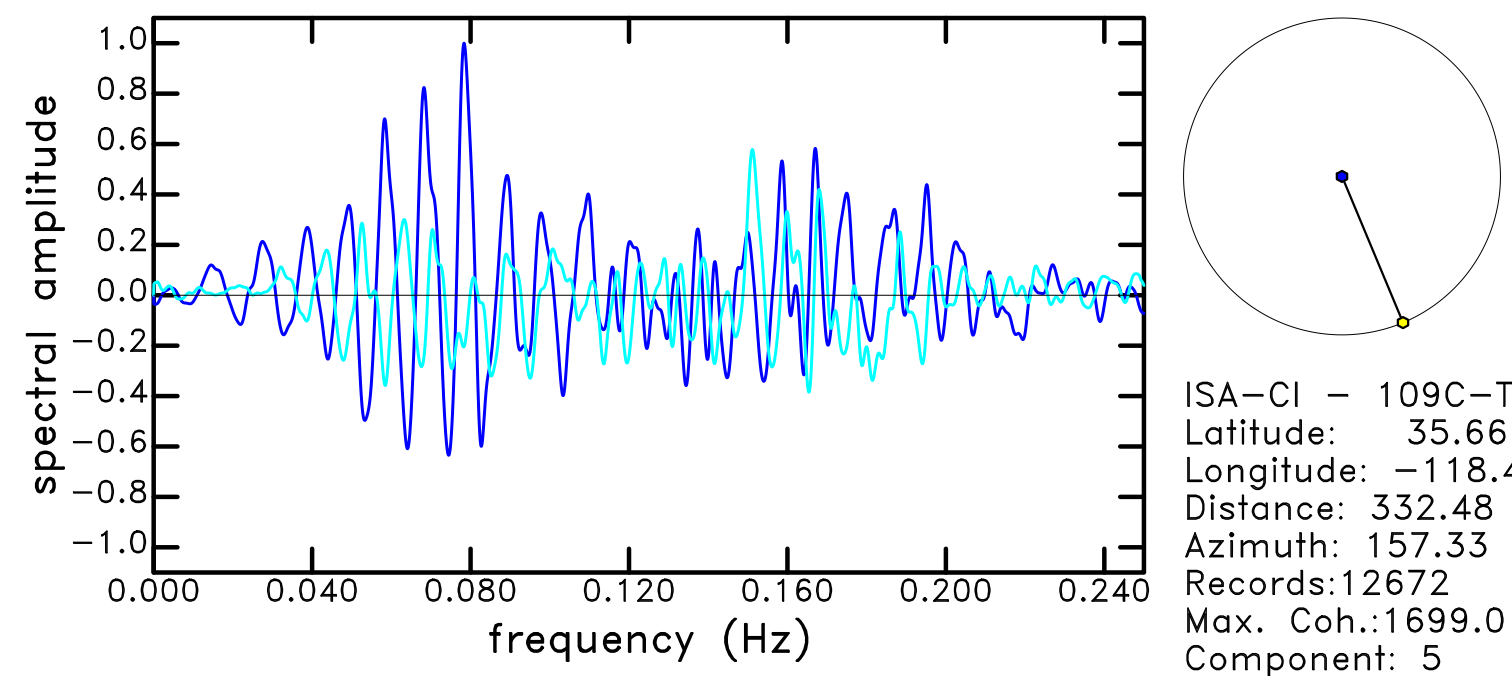

ISA-Cl - 109C-TA

Latitude: $\quad 35.66$

Longitude: -118.47

Distance: 332.48

Azimuth: 157.33

Records: 12672

Max. Coh.:1699.0

Component: 5

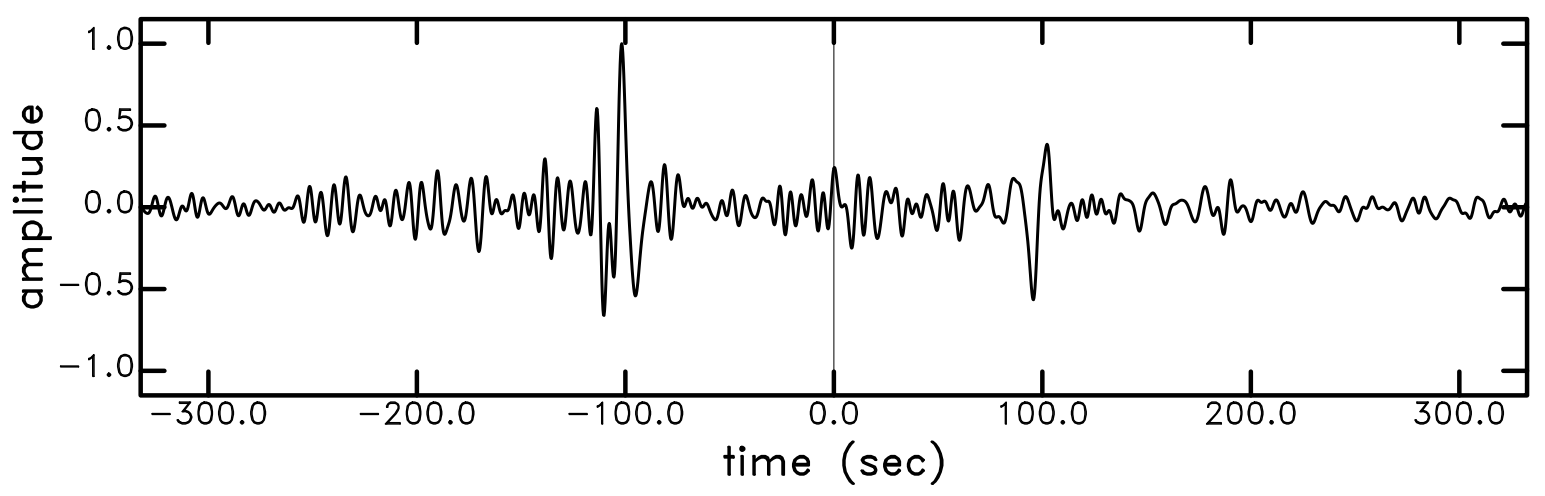

Figure 1: Transverse cross-correlation spectrum for stations ISA-CI and 109C-TA. The top panel shows the real (dark blue) and imaginary (light blue) components of the spectrum. Bottom panel shows the equivalent time-domain signal in the 300-s window centered on $0 \mathrm{~s}$ offset. 


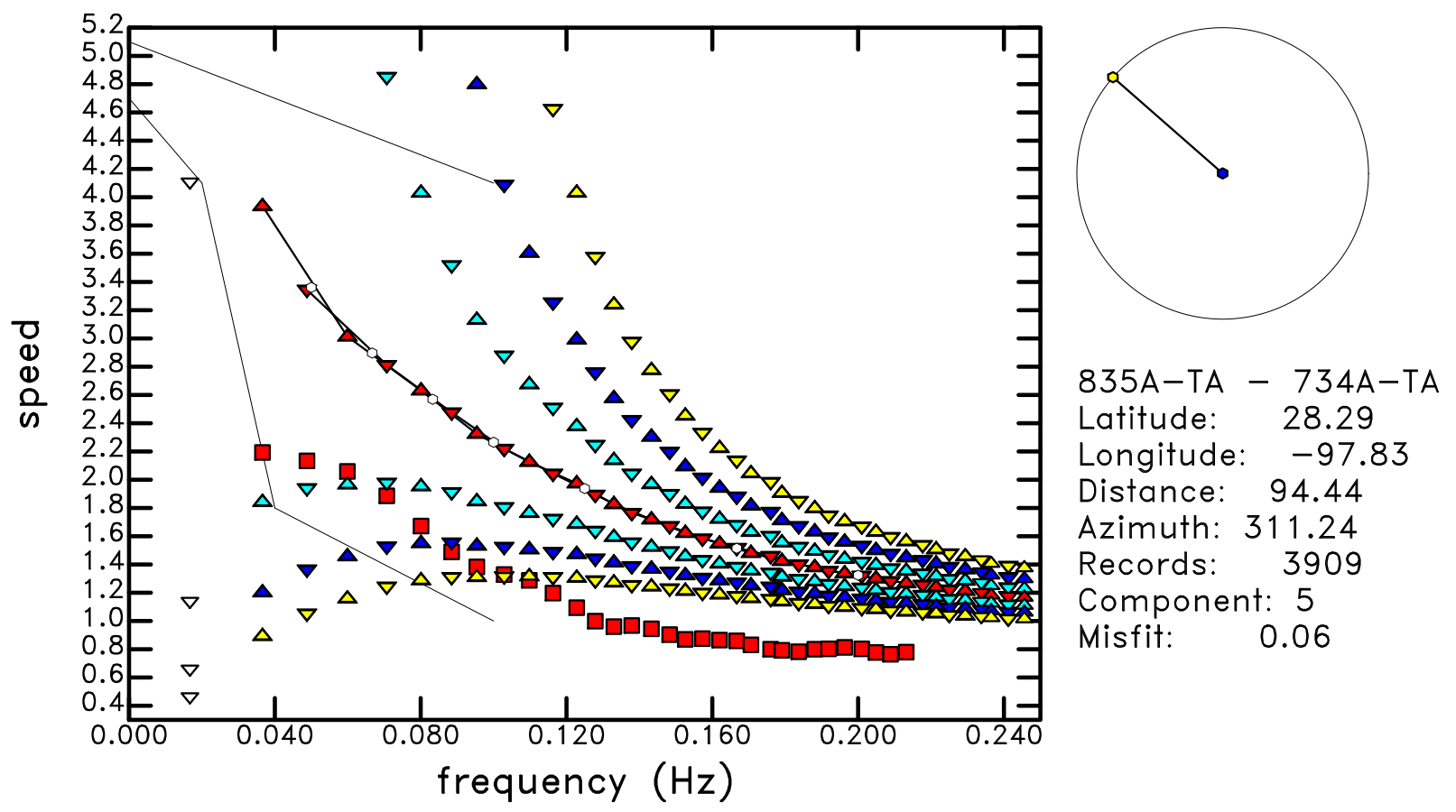

Figure 2: Diagram illustrating the construction of a Love wave dispersion curve from zero crossings of the cross-correlation spectrum for two stations in southern Texas. Triangles show frequency and phase speed corresponding to different branches $m$ in equation 4 . The thin lines show the acceptable range of phase velocities at low frequencies; only the red triangles fall in this range at 25 -s period, and this branch can be traced to 5 -sec period, defining the dispersion curve. Red squares show group velocities corresponding to this dispersion curve. 


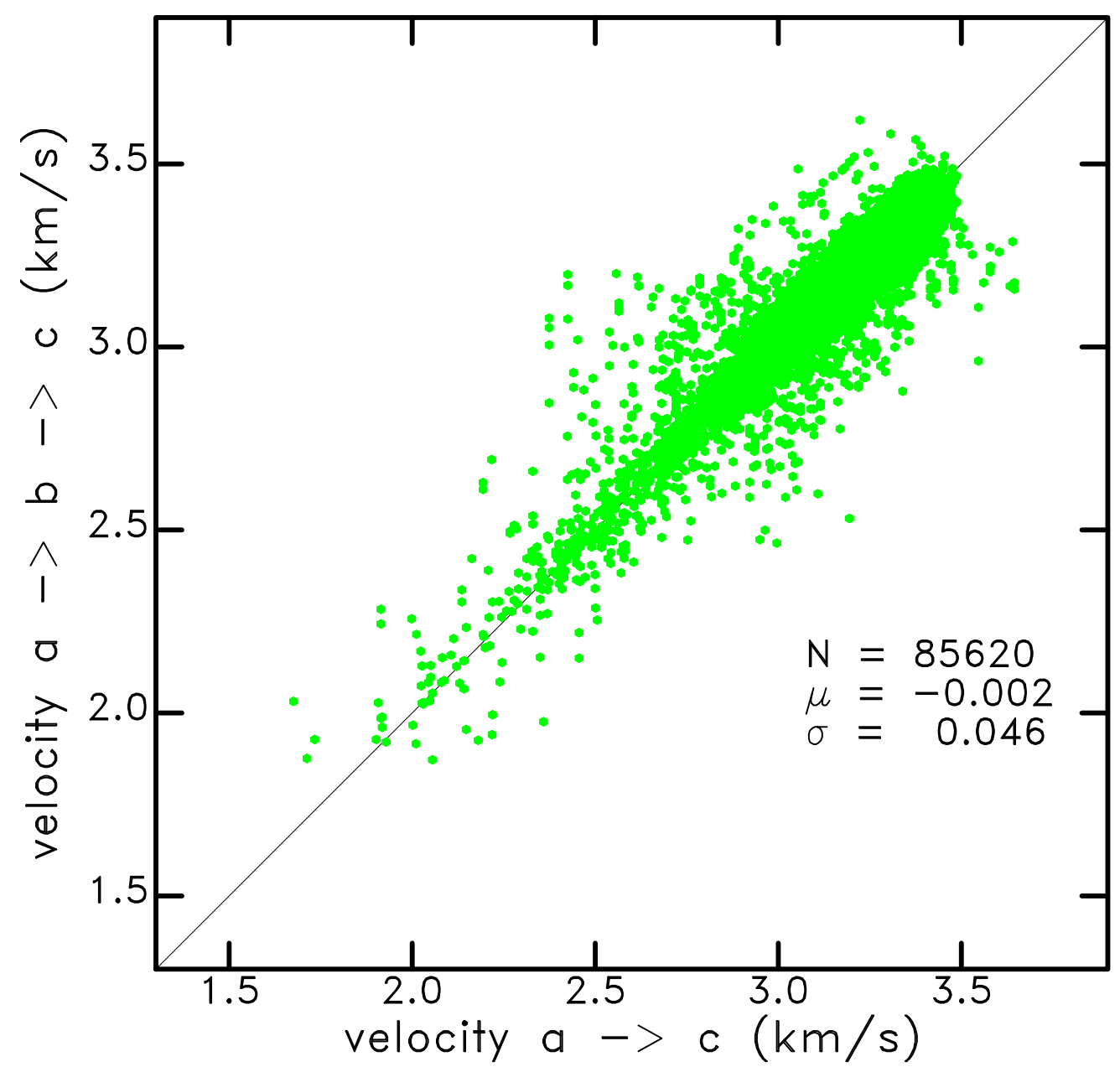

Figure 3: Scatter plot showing the comparison of phase-velocity observations for the path between stations $a$ and $c$. Horizontal axis gives the direct measurement $c_{a c}$. Vertical axis gives the measurement $c_{a c}^{\prime}$ obtained by combining two measurements: $a$ to $b$ and $b$ to $c$. 

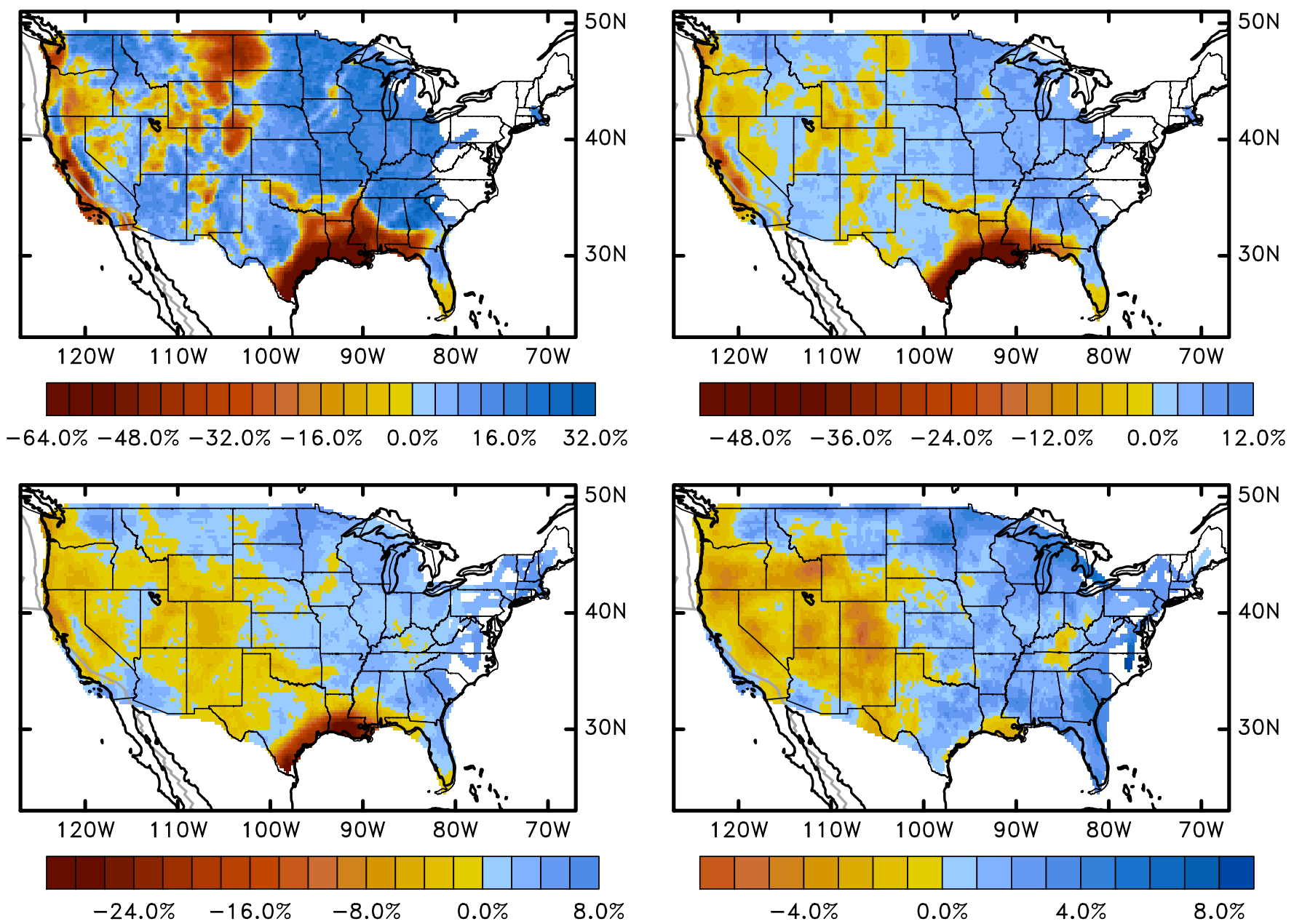

Figure 4: Maps of Love wave phase velocities at different periods: top left, $5 \mathrm{~s}$; top right, $10 \mathrm{~s}$; bottom left, $20 \mathrm{~s}$; and bottom right, $40 \mathrm{~s}$. The maps are parameterized using $0.25^{\circ}-$ by- $0.25^{\circ}$ pixels, and deviations are shown with respect to the mean (Table 1 ). Note the different scales at different periods, and the skewed distribution of deviations at short periods. 

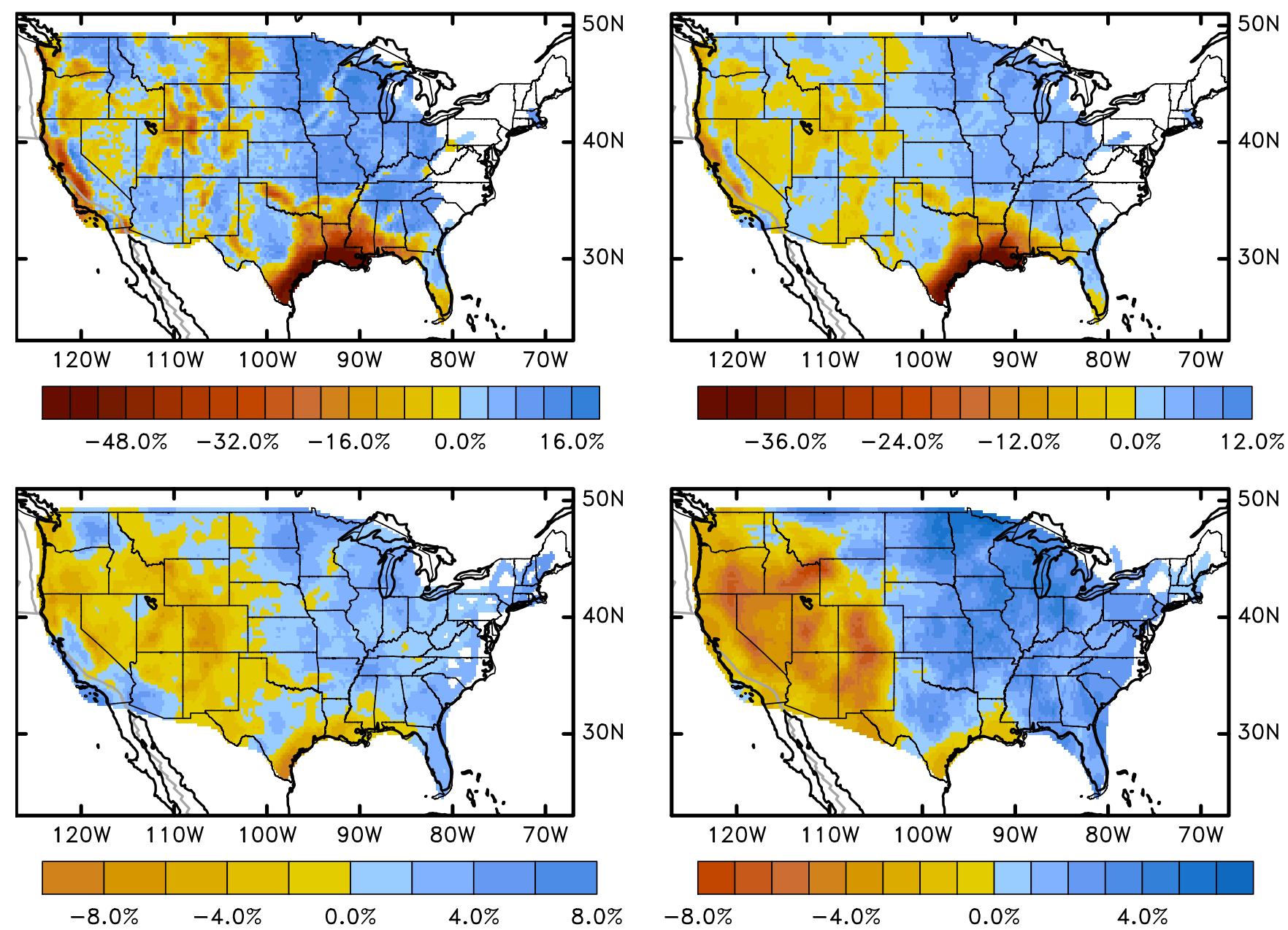

Figure 5: Maps of Rayleigh wave phase velocities at different periods: top left, 5 s; top right, $10 \mathrm{~s}$; bottom left, $20 \mathrm{~s}$; and bottom right, $40 \mathrm{~s}$. The maps are parameterized using $0.25^{\circ}$-by- $0.25^{\circ}$ pixels, and deviations are shown with respect to the mean (Table 1 ). Note the different scales at different periods, and the weaker expression of some short-period anomalies, as compared with the Love wave maps (Figure 4). 

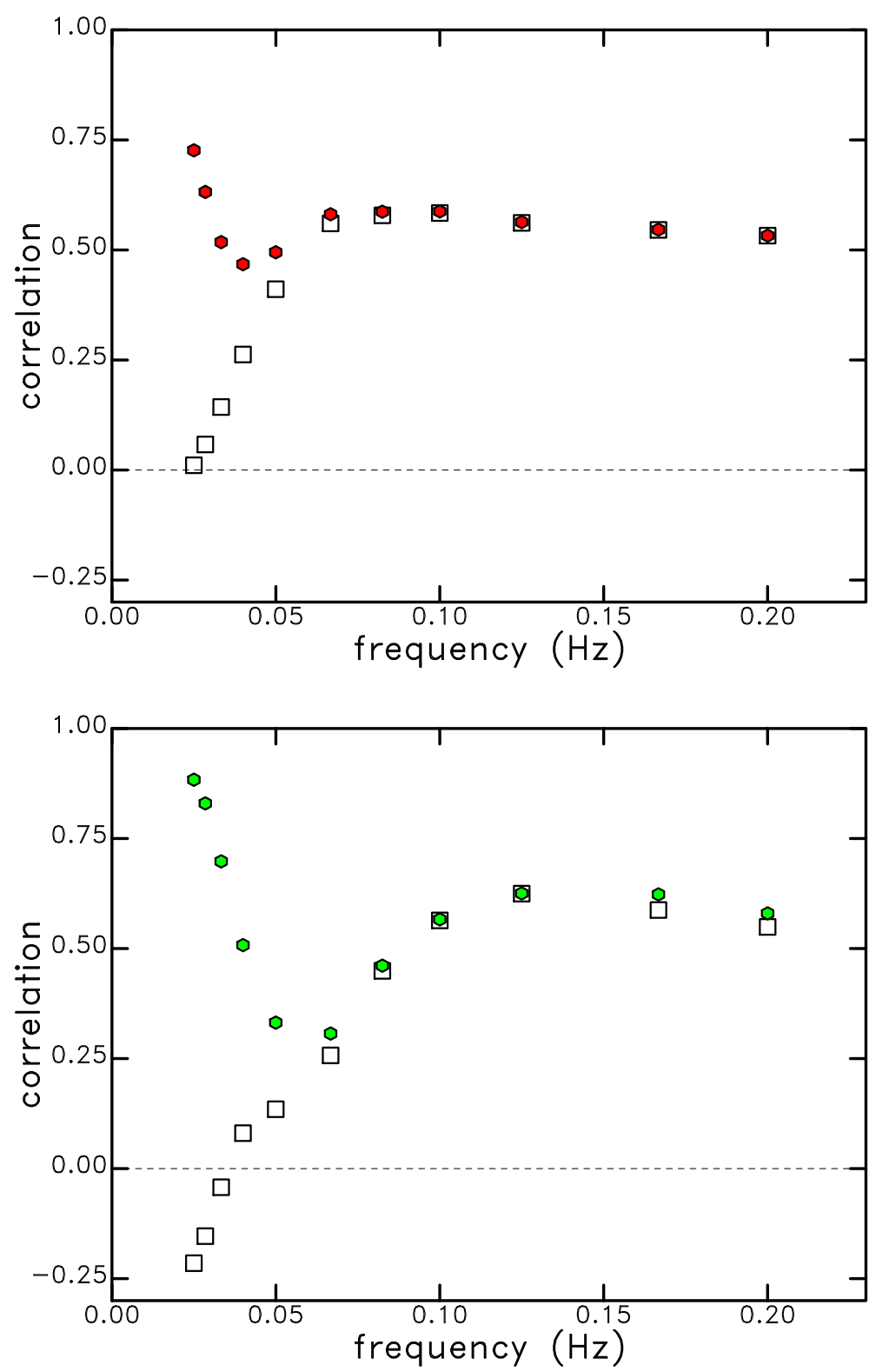

Figure 6: Correlation of observed and predicted phase-velocity maps. Open squares show correlation with predicted maps based on CRUST2.0 and the PREM mantle. Filled hexagons show correlations with predicted maps based on CRUST2.0 and the 3-D mantle model ND08. (top) Love waves. (bottom) Rayleigh waves. 

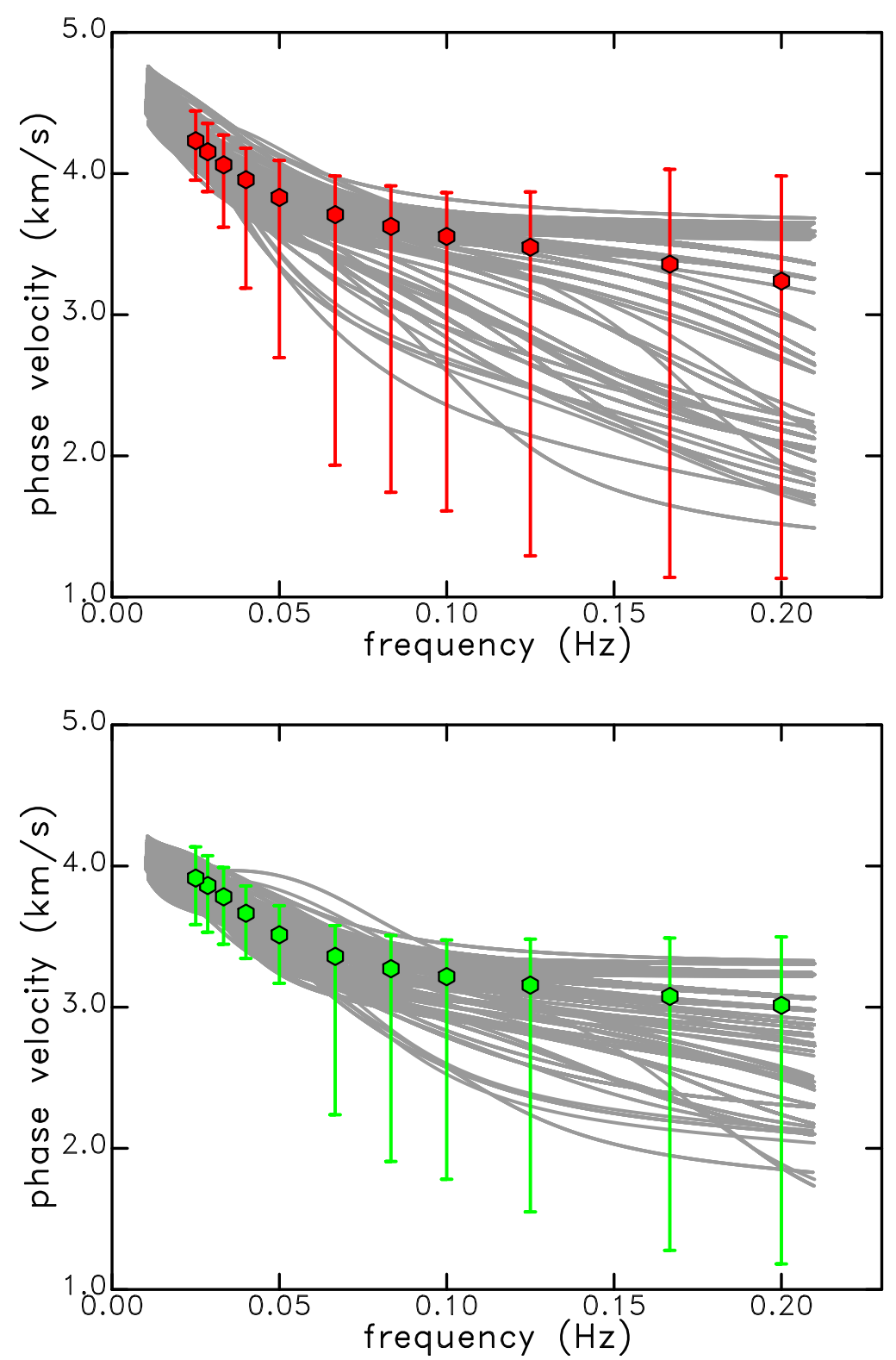

Figure 7: Comparison of observed phase-velocity ranges and those predicted by CRUST2.0 and ND08. (top) Median observed Love wave phase velocity (hexagons) at eleven different periods and the range of observed values (bars) at each period. (bottom) Same as top, but for Rayleigh waves. 

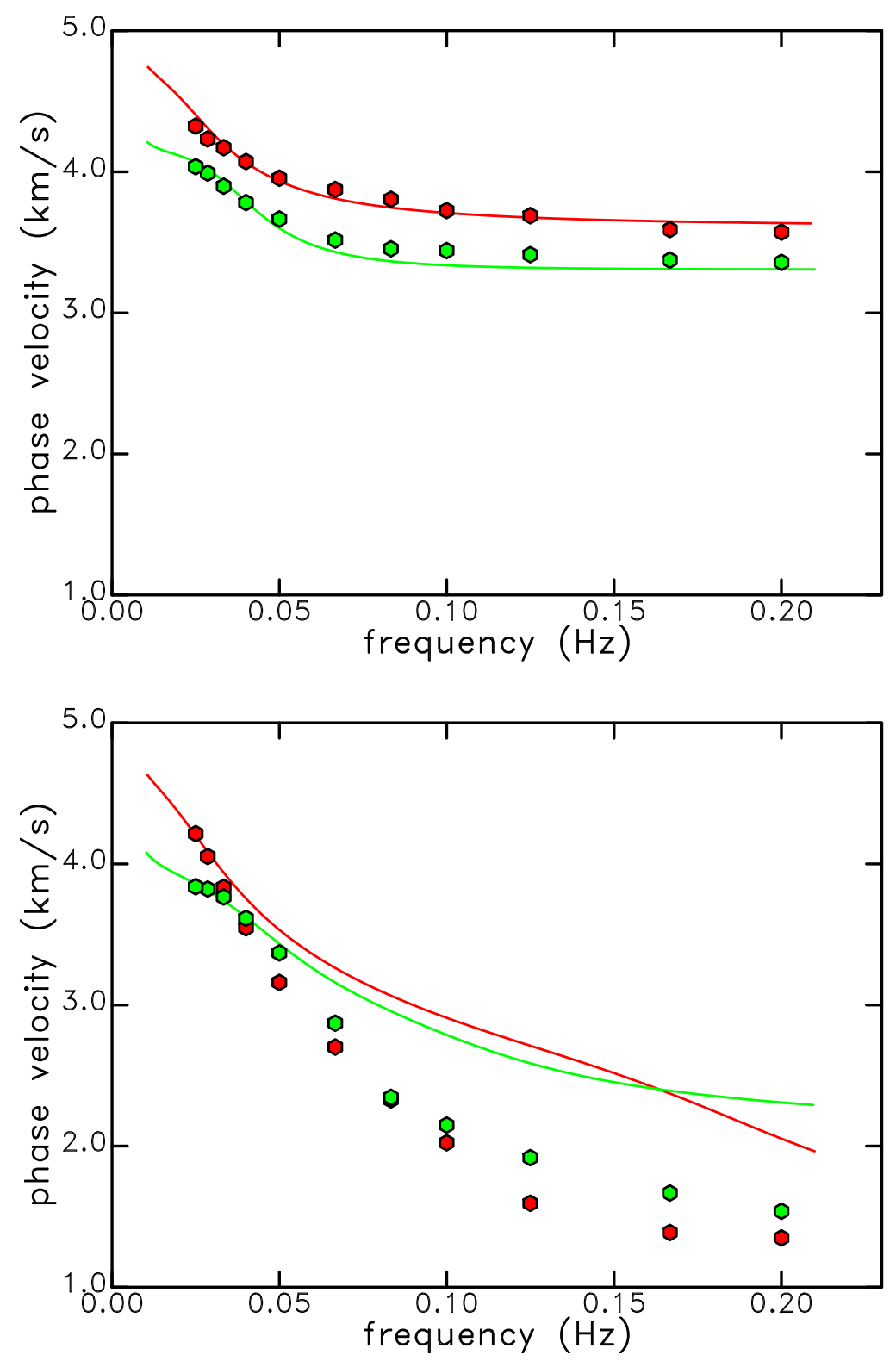

Figure 8: Comparison of observed (symbols) and predicted (lines) phase velocities. (top) Love (red) and Rayleigh (green) wave dispersion at $47^{\circ} \mathrm{N}, 95^{\circ} \mathrm{W}$ (northern Minnesota), showing good agreement. (bottom) Same as top, but for location $31^{\circ} \mathrm{N}, 91^{\circ} \mathrm{W}$ (southern Mississippi), showing large differences for periods shorter than $20 \mathrm{~s}$. 

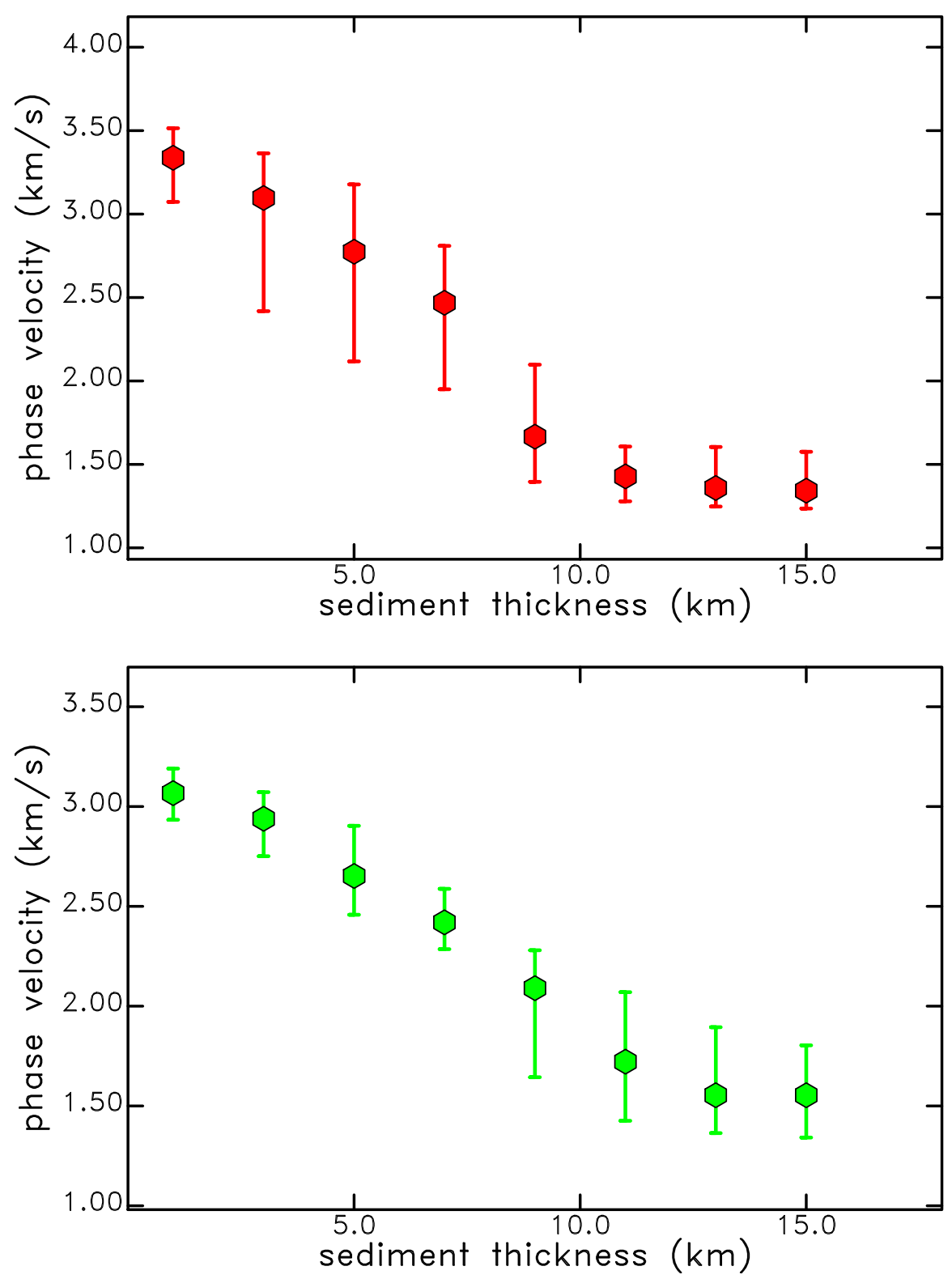

Figure 9: Phase velocities at 5-s period shown as a function of the thickness of the sedimentary cover. Symbols show the median value for all map pixels that correspond to a specific two-kilometer range of thicknesses, based on Mooney and Kaban (2010). The vertical bars show the range of the central two quartiles of the distribution of phase velocities for these pixels. (top) Love waves. (bottom) Rayleigh waves. 